\title{
AN ALGEBRAIC CHARACTERIZATION OF CERTAIN ORTHONORMAL SYSTEMS ${ }^{1}$
}

\section{J. J. PRICE}

1. Introduction. The system $\Phi=\left\{\phi_{n}\right\}_{0}^{\infty}$ of Haar functions is an orthonormal set in $L^{2}[0,1]$ with the following algebraic properties:

(a) $\phi_{0}(x) \equiv$ constant.

(b) If $n>k, \phi_{n} \phi_{k}=c_{n k} \phi_{n}$ where $c_{n k}$ is a constant, possibly zero.

(c) $\phi_{\mathfrak{n}}^{2}$ is a linear combination of $\phi_{0}, \phi_{1}, \cdots, \phi_{n-1}$.

In this paper, we prove that the above properties characterize orthonormal sets that are essentially certain subsets of the Haar system. In fact, (b) and (c) may be replaced by a more general assumption.

(d) For each $n \geqq 0$, the linear space spanned by $\left\{\phi_{0}, \phi_{1}, \cdots, \phi_{n}\right\}$ is a ring. These results follow from Theorem 1 , in which weaker assumptions are made and a wider class of orthonormal sets is characterized.

Generalizations and related results are given in $\S 4$.

2. Definitions and notation. All functions will be real-valued and belong to $L^{2}(X, \mu)$ where $(X, \mu)$ is a totally finite measure space.

$(\phi, \psi)$ will denote the usual inner product in $L^{2}(X, \mu)$.

For a given set of functions $\Phi, \Lambda(\Phi)$ will denote the set of all finite linear combinations of elements of $\Phi$.

For a given function $\phi$, the set where $\phi$ is nonzero will be denoted by $\sigma(\phi)$ and will be called the support of $\phi$.

If $P=\left\{X_{i}\right\}$ is a partition of $X$ into a finite number of subsets, or parts, of positive measure, $\nu(P)$ will be the number of parts. If $P$ and $P^{\prime}$ are partitions of $X$, we write $P^{\prime}<P$ if $P^{\prime}$ refines $P$ but $P^{\prime} \neq P$. I will denote the identity partition. A function constant on each part of a partition $P$ will be called a step function $(P)$.

Certain orthonormal systems will be called Haar sets (for details, see $[1],[2])$. Consider a sequence $\left\{P_{i}\right\}_{0}^{\infty}$ of partitions of the following type.

(i) $P_{0}=I$.

(ii) $P_{0}>P_{1}>P_{2}>\cdots \cdot$

(iii) $P_{i+1}$ is formed by splitting one part of $P_{i}$ into two new parts. Observe that (i) and (iii) imply $\nu\left(P_{i}\right)=i+1$. There is an orthonormal

Received by the editors December 20, 1966.

1 This work was supported by grants from the Institute for Advanced Study and the National Science Foundation. 
set $\left\{\phi_{i}\right\}_{0}^{\infty}$, unique except for choice of signs, such that $\phi_{0}$ is a constant, and for $i \geqq 1, \phi_{i}$ is constant on each of the new parts of $P_{i}$ and vanishes otherwise. Such an orthonormal system we call a Haar set. When $X$ is the unit interval and at each stage the two new parts have equal measures, the corresponding Haar set is a subsystem of the ordinary Haar functions. (Not every subsystem of the Haar functions, however, is a Haar set.)

The notion of a Haar set can be generalized in the following way. Let $\left\{P_{1}\right\}_{0}^{\infty}$ be a sequence of partitions such that

(iv) $P_{0}>P_{1}>P_{2}>\cdots, \nu\left(P_{i}\right)=n_{i}+1$ where $\left\{n_{i}\right\}_{0}^{\infty}$ is an increasing sequence of integers,

(v) $P_{i+1}$ is formed by splitting one part of $P_{i}$ into $n_{i+1}-n_{i}+1$ new parts.

There are orthonormal sets $\left\{\phi_{n}\right\}_{0}^{\infty}$ with the property that for each $i$, the functions $\phi_{n}, n_{i}<n \leqq n_{i+1}$, are constant on the new parts of $P_{i+1}$ and vanish otherwise $\left(n_{-1}=-1\right)$. These will be called generalized Haar sets.

We remark that it is meaningful to speak also of finite sets of this type.

3. The main theorem. We now characterize generalized Haar sets by certain algebraic properties less restrictive than (a), (b), and (c) of the introduction.

TheOREM 1. Let $\Phi=\left\{\phi_{n}\right\}_{0}^{\infty}$ be an orthonormal set in $L^{2}(X, \mu)$. Suppose

(1) $\phi_{0}(x)$ does not vanish.

(2) If $n>k, \phi_{n} \phi_{k} \in \Lambda\left(\phi_{0}, \phi_{1}, \cdots, \phi_{n}\right)$.

(3) For each $n, \phi_{n}^{2} \in \Lambda(\Phi)$. Then, after a suitable reordering, $\Phi$ is a generalized Haar set.

Proof. By hypothesis (2),

$$
\phi_{n} \phi_{k}=\sum_{\nu=0}^{n} a_{n k}^{(\nu)} \phi_{\nu} \quad \text { when } n>k .
$$

Let $j<n, j \neq k$.

$$
a_{n k}^{(j)}=\left(\phi_{n} \phi_{k}, \phi_{j}\right)=\left(\phi_{n}, \phi_{k} \phi_{j}\right)=\left(\phi_{n}, \sum_{\nu=0}^{l} a_{k j}^{(\nu)} \phi_{\nu}\right)=\sum_{\nu=0}^{l} a_{k j}^{(\nu)}\left(\phi_{n}, \phi_{\nu}\right)
$$

where $l=\max \{k, j\}<n$. By orthogonality, $a_{n k}^{(j)}=0$, and so the expansion (4) reduces to

$$
\phi_{n} \phi_{k}=a_{n k}^{(n)} \phi_{n}+a_{n k}^{(k)} \phi_{k} .
$$


We introduce an equivalence relation in to the set $\Phi$ as follows:

$$
\phi_{n} \approx \phi_{k} \equiv \phi_{n} \phi_{k}=a \phi_{n}+b \phi_{k}, \quad a b \neq 0 \quad(n \neq k) ; \quad \phi_{n} \approx \phi_{n} .
$$

The transitivity of this relation is easily checked. If $\phi_{n} \sim \phi_{k}$ and $\phi_{m} \sim \phi_{n}$, and if we assume no two of $n, m, k$ are equal, as we may, then

$$
\phi_{n} \phi_{k}=a \phi_{n}+b \phi_{k}, \quad \phi_{m} \phi_{n}=c \phi_{m}+d \phi_{n}
$$

where all coefficients are nonzero. Multiplying the first of the above relations by $\phi_{m}$, the second by $\phi_{k}$ and equating yields

$$
(b-c) \phi_{m} \phi_{k}=d \phi_{n} \phi_{k}-a \phi_{m} \phi_{n} .
$$

Substituting relations (6) in the above equation yields

$$
(b-c) \phi_{m} \phi_{k}=b d \phi_{k}-a c \phi_{m} .
$$

Both coefficients on the right-hand side above are nonzero. Therefore $b-c$ must be nonzero, and so $\phi_{m} \sim \phi_{k}$.

Let $\left[\phi_{n}\right]$ denote the equivalence class of $\phi_{n}$. We assert that each class $\left[\phi_{n}\right]$ is finite. If $\phi_{n} \sim \phi_{k}$, and $n \neq k$, then

$$
\left(\phi_{n}^{2}, \phi_{k}\right)=\left(\phi_{n}, \phi_{n} \phi_{k}\right)=\left(\phi_{n}, a_{n k}^{(n)} \phi_{n}+a_{n k}^{(k)} \phi_{k}\right)=a_{n k}^{(n)} \neq 0 .
$$

But hypothesis (3) guarantees that $\left(\phi_{n}^{2}, \phi_{k}\right)$ is nonzero for only a finite number of functions $\phi_{k}$. Therefore $\left[\phi_{n}\right]$ is finite.

If $\phi_{n}$ is not equivalent to $\phi_{k}$, there are three possibilities. Either $\phi_{n} \phi_{k}=a_{n k}^{(n)} \phi_{n}, \phi_{n} \phi_{k}=a_{n k}^{(\mathbf{k})} \phi_{k}$, or $\phi_{n} \phi_{k}=0$. We now make the following assumptions about the enumeration of our system $\Phi$.

(7) If $\phi_{n} \phi_{k}=a_{n k}^{(n)} \phi_{n}$, where $a_{n k}^{(n)} \neq 0$, then $n>k$.

(8) The members of each equivalence class are consecutively indexed. It will be shown later that $\Phi$ can be reordered so that (7) and (8) are satisfied.

Because of (8), there is an increasing sequence of integers $\left\{n_{i}\right\}_{-1}^{\infty}$, $n_{-1}=-1$, such that each equivalence class is a set of the form $\left\{\phi_{j}: n_{i}<j \leqq n_{i+1}\right\}$.

Let $\Lambda_{n}=\Lambda\left(\phi_{0}, \phi_{1}, \cdots, \phi_{n}\right)$. We claim $\Lambda_{n_{i}}$ is an algebra over the reals. It suffices to show $\phi_{j} \phi_{k} \in \Lambda_{n_{i}}$ whenever $\phi_{j}, \phi_{k} \in \Lambda_{n_{i}}$. For $j \neq k$, this is so by hypothesis (2). $\phi_{k}^{2} \in \Lambda_{n_{i}}$ if $\phi_{k}^{2}$ is orthogonal to $\phi_{r}$ whenever $r>n_{i}$. Now $\Lambda_{n_{i}}$ contains [ $\left.\phi_{k}\right]$. Therefore, $\phi_{k}$ is not equivalent to $\phi_{r}$, and so, using (7),

$$
\left(\phi_{k}^{2}, \phi_{r}\right)=\left(\phi_{k}, \phi_{r} \phi_{k}\right)=a_{r k}^{(r)}\left(\phi_{k}, \phi_{r}\right)=0 .
$$

Hence $\phi_{\boldsymbol{k}}^{2} \in \Lambda_{n_{i}}$.

We conclude that $\Lambda(\Phi)$ is an algebra of the form $\cup_{i=0}^{\infty} \Lambda_{n_{i}}$ where $\Lambda_{n_{i}}$ is a subalgebra with orthonormal basis $\left\{\phi_{0}, \phi_{1}, \cdots, \phi_{n_{i}}\right\}$. 
Consider a fixed subalgebra $\Lambda_{n_{i}}$. If $\phi_{k} \in \Lambda_{n_{i}}$, then since the dimension of $\Lambda_{n_{i}}$ is $n_{i}+1$, the functions $\phi_{k}, \phi_{k}^{2}, \cdots, \phi_{k}^{n_{i}+2}$ are linearly dependent. Therefore $\phi_{k}$ can assume only a finite number of values. Consequently, $\Lambda_{n_{i}}$ induces a finite number of separation classes in $X$, and may be regarded as an algebra of continuous real functions that separate points of a finite (compact) space $X^{\prime}$ and do not all vanish at any given point. By an elementary form of the Stone-Weierstrass Theorem, $\Lambda_{n_{i}}$ is the set of all real functions on $X^{\prime}$, and card $X^{\prime}$ $=\operatorname{dim} \Lambda_{n_{i}}=n_{i}+1$. In other words, $\Lambda_{n_{i}}$ is the space of all step functions $\left(P_{i}\right)$ where $P_{i}$ is the partition of $X$ into separation classes induced by $\Lambda_{n_{i}}$ and $\nu\left(P_{i}\right)=n_{i}+1$.

Since $\Lambda_{n_{i}} \subset \Lambda_{n_{i+1}}$, the separation classes of $\Lambda_{n_{i+1}}$ are subsets of those of $\Lambda_{n_{i}}$. Hence $P_{i+1}<P_{i}$. In order to establish that $\Phi$ is a generalized Haar set, it will suffice to show that all elements of $\Lambda_{n_{i+1}}-\Lambda_{n_{i}}$ vanish on each part of $P_{i}$ except one, for it will then follow that only one part of $P_{i}$ splits when $P_{i+1}$ is formed. The set $\Lambda_{n_{i+1}}-\Lambda_{n_{i}}=\left\{\phi_{k}: n_{i}<k \leqq n_{i+1}\right\}$ is an equivalence class. Since equivalent functions have identical supports, as is evident from (5), all we need prove is that the support of any of these functions $\phi_{k}$ is contained in one part of $P_{i}$.

Such a function $\phi_{k}$ is not equivalent to any member of $\Lambda_{n_{i}}$. Therefore, if $\phi_{j} \in \Lambda_{n_{i}}, \phi_{k} \phi_{j}=a_{k j}^{(k)} \phi_{k}$ (where $a_{k j}^{(k)}$ may be zero). By linearity, for each $\chi \in \Lambda_{n_{i}}, \phi_{k} \chi=c \phi_{k}$ where $c$ is a constant depending on $\chi$. Hence $\chi$ is constant on $\sigma\left(\phi_{k}\right)$. Since $\Lambda_{n_{i}}$ is the space of all step functions $\left(P_{i}\right)$, we may choose for $\chi$ the characteristic function of any part of $P_{i}$, say $X_{i 0}$, that intersects $\sigma\left(\phi_{k}\right)$. Since $\chi$ is constant on $\sigma\left(\phi_{k}\right)$ and $\chi(x)=1$ for some $x \in \sigma\left(\phi_{k}\right), \chi(x) \equiv 1$ on $\sigma\left(\phi_{k}\right)$. Hence $\sigma\left(\phi_{k}\right) \subset X_{i 0}$. Therefore $\Phi$ is a generalized Haar set.

It remains to be shown that there is a reordering of $\Phi$ such that the rearranged set $\Psi=\left\{\psi_{n}\right\}_{0}^{\infty}$ satisfies (7) and (8).

Equivalent elements of $\Phi$ have identical supports. If $\phi_{n}$ is not equivalent to $\phi_{k}$, then either

$$
\sigma\left(\phi_{n}\right) \subset \sigma\left(\phi_{k}\right), \quad \sigma\left(\phi_{k}\right) \subset \sigma\left(\phi_{n}\right), \text { or } \sigma\left(\phi_{n}\right) \cap \sigma\left(\phi_{k}\right)=\varnothing .
$$

These relations correspond to the cases

$$
\phi_{n} \phi_{k}=a_{n k}^{(n)} \phi_{n}, \quad \phi_{n} \phi_{k}=a_{n k}^{(k)} \phi_{k}, \quad \phi_{n} \phi_{k}=0 .
$$

Introduce a partial ordering in to the set $[\Phi]$ of equivalence classes as follows. Define $\left[\phi_{n}\right] \prec\left[\phi_{k}\right]$ if $\sigma\left(\phi_{n}\right) \subset \sigma\left(\phi_{k}\right)$. Otherwise let $\left[\phi_{n}\right]$ and $\left[\phi_{k}\right]$ be not comparable. This is obviously a well-defined partial ordering.

Suppose $\left[\phi_{n}\right] \prec\left[\phi_{k}\right]$. Then 


$$
\left(\phi_{n}^{2}, \phi_{k}\right)=\left(\phi_{n}, \phi_{n} \phi_{k}\right)=\left(\phi_{n}, a_{n k}^{(n)} \phi_{n}\right)=a_{n k}^{(n)} \neq 0 .
$$

We have already pointed out that $\left(\phi_{n}^{2}, \phi_{k}\right)$ is nonzero for only a finite number of functions $\phi_{k}$. Hence $\left[\phi_{n}\right] \prec\left[\phi_{k}\right]$ for only a finite number of classes $\left[\phi_{k}\right]$. It follows that every ordered chain in $[\Phi]$ has a maximal element and the same is true if a finite number of elements are deleted from $[\Phi]$. Therefore, by Zorn's Lemma, these partially ordered sets have maximal elements.

Enumerate $[\Phi]$ as follows. As first element choose $\left[\phi_{0}\right]$, the unique maximal element of $[\Phi]$. Next, from the remaining equivalence classes choose the maximal element that contains $\phi_{k}$ with minimal $k$. Continuing in this way, we obtain an ordering in $[\Phi]$.

Using the ordering in $[\Phi]$ in an obvious manner, we can obtain an ordering $\Psi=\left\{\psi_{n}\right\}_{0}^{\infty}$ of $\Phi$ so that $\psi_{0}=\phi_{0}$ and the members of each equivalence class are assigned consecutive indices. Such a rearrangement has the desired properties for if $\psi_{n} \psi_{k}=c_{n k}^{(n)} \psi_{n}$ where $c_{n k}^{(n)} \neq 0$, then $\left[\psi_{n}\right] \prec\left[\psi_{k}\right]$ which implies $n>k$.

This completes the proof of Theorem 1.

4. Related results. Orthonormal sets with properties (a) and (d) of the introduction satisfy the hypotheses of Theorem 1 . Hence, they are generalized Haar sets with $n_{i}=i$. Therefore, we obtain the following corollary.

THEOREM 2. Haar sets are characterized among orthonormal systems by properties (a) and (d).

The techniques used in the proof of Theorem 1 can be applied to obtain the following result.

Theorem 3. Let $\Phi=\left\{\phi_{n}\right\}_{0}^{\infty}$ be an orthonormal set in $L^{2}(X, \mu)$. Suppose there is an increasing sequence $\left\{n_{i}\right\}_{0}^{\infty}$ of integers such that $\Lambda_{n_{i}}$ is a ring, $i \geqq 0$. Then there exists a decreasing sequence $\left\{P_{i}\right\}_{0}^{\infty}$ of partitions of $X$ such that for each $i \geqq 0, \nu\left(P_{i}\right)=n_{i}+1$ and $\Lambda_{n_{i}}$ is the set of all step functions $\left(P_{i}\right)$.

The converse of Theorem 3 is obviously true.

There is a connection between Theorem 3 and a previous result of the author. Let $\Phi=\left\{\phi_{n}\right\}_{0}^{\infty}$ be an orthonormal set in $L^{2}(X, \mu), \phi_{0}(x)$ $\equiv$ constant. Associated with $\Phi$ are the Dirichlet kernels

$$
D_{n}(x, y)=\sum_{\nu=0}^{n-1} \phi_{\nu}(x) \phi_{\nu}(y) .
$$

In [3] it is shown that the conclusion of Theorem 3 above is necessary and sufficient for the kernels $D_{n_{i}+1}(x, y)$ to be nonnegative, $i \geqq 0$. Therefore, we have the following theorem. 
Theorem 4. Let $\left\{\phi_{n}\right\}_{0}^{\infty}$ be an orthonormal set in $L^{2}(X, \mu), \phi_{0}(x)$ $\equiv$ constant. There exists a sequence $\left\{D_{n_{i}}(x, y)\right\}_{0}^{\infty}$ of nonnegative kernels . if and only if $\Lambda_{n_{i}-1}$ is a ring, $i \geqq 0$.

We conclude by studying the effect of hypothesis (1) in Theorem 1.

THEOREM 5. Suppose all assumptions of Theorem 1 hold except (1). Then there is a countable family $\left\{X_{j}\right\}$ of pairwise disjoint subsets of $X$ such that the support of each function $\phi_{n}$ is contained in one of the sets $X_{j}$. Let

$$
\Phi_{j}=\left\{\phi \in \Phi: \sigma(\phi) \subset X_{j}\right\} .
$$

Each $\Phi_{j}$ can be reordered so that the rearranged set $\Psi_{j}$ is a generalized Haar set.

Proof. Reorder $\Phi$ as in Theorem 1. Then, if $n>k$, either $\psi_{n} \sim \psi_{k}$, $\psi_{n} \prec \psi_{k}$, or $\psi_{n}$ and $\psi_{k}$ are not comparable. In terms of supports, either $\sigma\left(\psi_{n}\right)=\sigma\left(\psi_{k}\right), \sigma\left(\psi_{n}\right) \subset \sigma\left(\psi_{k}\right)$ or, $\sigma\left(\psi_{n}\right)$ and $\sigma\left(\psi_{k}\right)$ are disjoint.

Define $X_{0}=\sigma\left(\psi_{0}\right)$. Let $\psi_{k_{1}}$ be the first element of $\Psi$, if any exists, whose support is disjoint from $X_{0}$ and define $X_{1}=\sigma\left(\psi_{k_{1}}\right)$. Let $\psi_{k_{2}}$ be the first element whose support is disjoint from $X_{0} \cup X_{1}$ and define $X_{2}=\sigma\left(\psi_{k_{2}}\right)$. Continuing in this way, we obtain a finite or denumerably infinite family $\left\{X_{j}\right\}$ of pairwise disjoint subsets of $X$. Every element of $\Psi$ belongs to one of the sets

$$
\Psi_{j}=\left\{\psi \in \Psi: \sigma(\psi) \subset X_{j}\right\} .
$$

After an obvious renumbering, the system $\Psi_{j}$ satisfies, relative to $X_{j}$, conditions (2) and (3) of Theorem 1. Condition (1) is satisfied also since $X_{j}=\sigma\left(\psi_{k j}\right)$. Therefore $\Psi_{j}$ is a generalized Haar set and the proof is complete.

We remark that some of the sets $\Psi_{j}$ may be finite. But Theorem 1 obviously applies to finite orthonormal sets as well as to infinite ones.

Theorem 5 can be stated algebraically. For each $j, \Psi_{j}$ contains the characteristic function of $X_{j}$. Therefore the ring $\Lambda(\Psi)$ contains a set $\left\{e_{j}\right\}$ of mutually orthogonal idempotents and splits into the direct sum of ideals $e_{j} \Lambda(\Psi)=\Lambda\left(\Psi_{j}\right)$.

\section{REFERENCES}

1. A. Haar, Zur Theorie der orthogonal Funktionensysteme, Math. Ann. 69 (1910), 331-371.

2. J. J. Price, Orthonormal sets with nonnegative Dirichlet kernels, Trans. Amer. Math. Soc. 95 (1960), 256-62.

3. - Orthonormal sets with nonnegative Dirichlet kernels. II, Trans. Amer. Math. Soc. 100 (1961), 153-61.

Purdue University 